Research Article

\title{
Prelabour rupture of membranes at term prospective study of expectant management versus induction of labour
}

\author{
Vijay Zutshi, Sumitra Bachani, Sakshi Goel*, Renu Arora, Sana Tiwari
}

Department of Obstetrics and Gynaecology, VMMC and SJH, New Delhi, India

Received: 01 May 2016 Accepted: 03 June 2016

\section{*Correspondence to:}

Dr. Sakshi Goel,

Email: doc.sakshig@gmail.com

Copyright: (C) the author(s), publisher and licensee Medip Academy. This is an openaccess article distributed under the terms of the Creative Commons Attribution NonCommercial License, which permits unrestricted noncommercial use, distribution, and reproduction in any medium, provided the original work is properly cited.

\begin{abstract}
Background: Premature rupture of membranes (PROM) complicates 5-10\% of pregnancies. Approximately 60-70 \% of term PROM cases are followed by the onset of labor within 24 hours. Diagnosis and proper management is very important. In spite of many studies available in the literature, the clinical management is surprisingly controversial.
\end{abstract}

Methods: Study conducted was prospective randomised controlled trial. Total 150 women were selected fulfilling the inclusion criteria, randomly allotted to the 3 groups. In group A, patients were observed for 24 hours. If labor didn't supervene in 24 hours since admission, induction of labor was done depending on the bishop's score. In the group B, labour was induced by vaginal misoprostol 25 micrograms given 4 hourly for 4 doses and in group C, labor was induced by instillation of $0.5 \mathrm{mg}$ PGE2 gel in the posterior fornix. The women were observed for onset and progress of labour. Failure of induction was considered if patient was not in established labour within 24 hours of instillation of first dose of cerviprime/misoprostol. Labour was monitored and managed as per hospital protocol. The analysis verified the following variables: duration of latent phase and active phase of labour, mode of delivery (spontaneous/vaccum/forceps/LSCS), third stage complications (PPH/fever/retained placenta), neonatal outcome.

Results: Thirty percent women had onset of spontaneous labor during expectant management in group A. The durations of latent phase and active phase of labour were lower in group B and C than group A (9 and 10.4 versus 15 hours; $\mathrm{p}<0.001$ ) and (4 and 6 versus10 hours; $\mathrm{p}<0.001$ ), respectively. Immediate induction in group $\mathrm{B}$ and $\mathrm{C}$ resulted in significantly lower rate of caesarean section $(17 \%$ and $19 \%$ versus $28.5 \%, \mathrm{P}=0.049)$ and operative vaginal delivery (5\% and $3 \%$ versus $13 \%, \mathrm{P}=0.007$ ). Only a few maternal-neonatal infections occurred and no significant difference was noted $(2.7 \%$ and $3 \%$ versus $3.5 \%, \mathrm{P}=$ $0.71)$.

Conclusions: Immediate induction with prostaglandin shortens the delivery interval and lowers the caesarean section rate as compared to expectant management; however the neonatal outcome is similar in the three groups.

Keywords: Premature rupture of membranes, Expectant management, Induction, Obstetric outcome, Neonatal outcome

\section{INTRODUCTION}

Premature rupture of membranes (PROM) is defined as rupture of membranes before onset of labor and complicates $5-10 \%$ of pregnancies. At least $80 \%$ of cases of PROM occur at term. In spite of many studies available in the literature, the clinical management is surprisingly controversial. ${ }^{1}$
Approximately $60-70 \%$ of term PROM cases are followed by the onset of labor within $24 \mathrm{~h}$ and an additional 20-30\% will start within 72 hours. ${ }^{2,3}$ Diagnosis and proper management is very important as it is implicated for various fetal and maternal complications generally due to infection. There is a controversy as to whether patients should be kept on conservative management or induction should be carried out. The ACOG guidelines define "the waiting time as an adequate 
time for the latent phase of labour to progress" but the waiting time is not specified in terms of hours.4 Waiting time from PROM to spontaneous onset of labour has been reported by various studies as 96 hours, 48 hours, 24 hours or 18 hours. ${ }^{5-10}$ As per the Cochrane review there is no substantial difference in the induction and expectant management group regarding the maternal-neonatal outcomes and women should be informed on the risks and benefits of each option to be able them to make an informed choice. ${ }^{11}$

Induction has been tried with oxytocin or prostaglandins depending on the bishop's score. We compared PG E1 (misoprostol) and PG E2 (dinoprostone) for induction of labour in pre-labour rupture of membranes at term with expectant management. There are no published studies comparing PG E1 and PG E2 with expectant management to the best of our knowledge.

\section{METHODS}

A prospective case control study conducted after ethical committee approval in the Department of Obstetrics and Gynaecology in Vardhman Mahavir Medical College and Safdarjung hospital for six months. One hundred and fifty women were selected as per inclusion and exclusion criteria mentioned below and were assigned into three groups following randomization.

\section{Inclusion criteria}

- $\quad$ Patients of PROM with 37 to 40 weeks of gestation

- Single live fetus in cephalic presentation

- Bishops score of less than six

- Patient not in labour.

\section{Exclusion criteria}

- $\quad$ ROM more than 24 hours

- Patients with features of chorioamnionitis

- Fetal compromise

- Previous caesarian section

- History of antepartum hemorrhage

- Maternal medical diseases

- Moderate to Severe IUGR

- Absolute indications for caesarean section

- Gravida 4 and above.

\section{Diagnosis of PROM was based on}

- Clinical history of passage of liquor

- Pooling of fluid in posterior fornix as seen by speculum examination

- Per vaginum examination and

- $\quad$ Reduced liquor volume on sonography (AFI <5) in selected women where clinical findings were inconclusive.
At the time of diagnosis of rupture of membranes, bishop's score was recorded, following which patients were monitored for one hour to determine onset of labour and fetal wellbeing. If the woman was not in labour she was randomly allotted to either of the groups as per the lot. Prophylactic antibiotic of a penicillin group was given.

In group A, patients were observed for 24 hours. Monitoring was done by

- Temperature recording every 4 hours

- Fetal heart rate monitoring every hour

- No digital vaginal examination until the woman was clinically in active labor, and

- Induction if signs of chorioamnionitis developed.

The criteria for diagnosing chorioamnionitis were temperature more than $38{ }^{\circ} \mathrm{C}$ with any two of the five features, viz. maternal tachycardia, fetal tachycardia, uterine tenderness, foul discharge and maternal leukocytosis. If labor did not supervene in 24 hours since admission, induction of labor was done depending on the bishop's score.

In the group $\mathrm{B}$, labour was induced by vaginal misoprostol 25 micrograms given 4 hourly for maximum four doses and in group $\mathrm{C}$ labour was induced by instillation of $0.5 \mathrm{mg}$ PGE2 gel in the posterior fornix. The woman was observed for onset and progress of labour. If labor had not established within 6 hours, application of PGE2 gel was repeated (maximum two doses).

Failure of induction was considered if patient was not in established labour within 24 hours of instillation of first dose of cerviprime/misoprostol. Labour was monitored and managed as per hospital protocol.

\section{The outcomes of this study was}

\section{Maternal outcome}

- Time to onset of active labour

- Duration of labour

- Development of chorioamnionitis

- Presence of meconium stained liquor

- Spontaneous vaginal delivery

- Operative vaginal delivery

- Caesarean section.

Neonatal outcome

- Apgar score

- Resuscitation with oxygen

- Ventilation after initial resuscitation

- Stay in nursery ICU if required

- Neonatal infection. 


\section{Statistical analysis}

Data was recorded on a predesigned proforma and deciphered at the end of the study. Data was expressed as Mean $\pm 2 S D \quad(95 \%$ confidence intervals), numbers (percentages) and median and was analysed using Pearson's chi square/Fisher's exact test, repeated measures ANOVA (parametric or nonparametric) or multiple logistic regression techniques, whichever was appropriate depending upon their nature. In all cases, $p$ value $<0.05$ was considered significant. The then available Excel (Microsoft, Redmond, WA, USA) and SPSS version (IBM, Armonk, NY, USA) were used for data presentation and statistical analysis.

\section{RESULTS}

All subjects were between 19-28 years of age. Patients in the 3 groups were similar with respect to parity, previous history of PROM, previous history of abortions. In group A, $30 \%$ women went into spontaneous labour without augmentation of which $9(60 \%)$ delivered spontaneously. The latent phase in this group was 15 hours. And 6 (40\%) delivered by caesarean section or operative vaginal delivery. $70 \%$ of patients required augmentation of which $13(37 \%)$ delivered spontaneously and 22 (63\%) delivered by caesarean section or operative vaginal delivery. As shown in Figure 1, duration of latent phase of labour was significantly shorter in induction group- 9 hours in group $\mathrm{B}$ and 10.4 hours in group $\mathrm{C}$ as compared to the expectant management group.

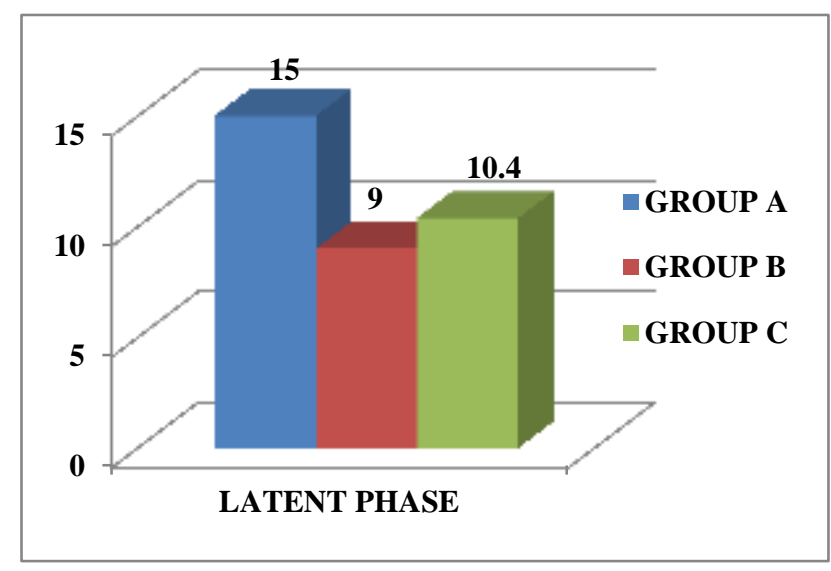

Figure 1: Duration of latent phase of labour.

- Comparing group A and B-p value $=<0.0001$.

- Comparing group A and C-p value $=<0.0001$.

As shown in Figure 2, duration of active phase of labour was significantly shorter in induction group- 4 hours in group $\mathrm{B}$ and 6 hours in group $\mathrm{C}$ as compared to 10 hours in expectant management group.

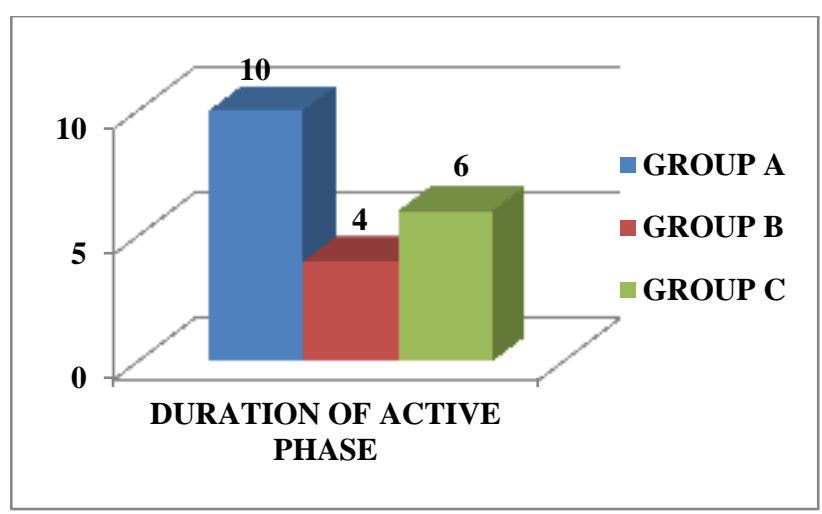

Figure 2: Duration of active phase of labour.

- Comparing group A and B-p value $=<0.0001$.

- Comparing group A and C- $\mathrm{p}$ value $=<0.0002$.

Presence of meconium stained liquor was higher in group B (22\%) compared to group A (14\%) and group C (12\%) but the difference was not statistically significant (Figure 3).

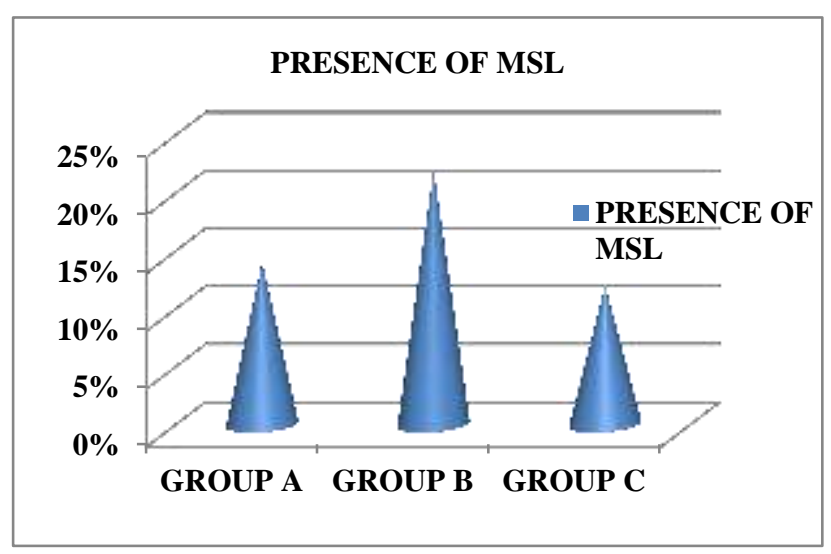

Figure 3: Presence of meconium stained liquor (MSL)

Presence of chorioamnionitis was higher in group A (4\%) as compared to the induction group $\mathrm{B}$ and $\mathrm{C}$ but the difference is not statistically significant.

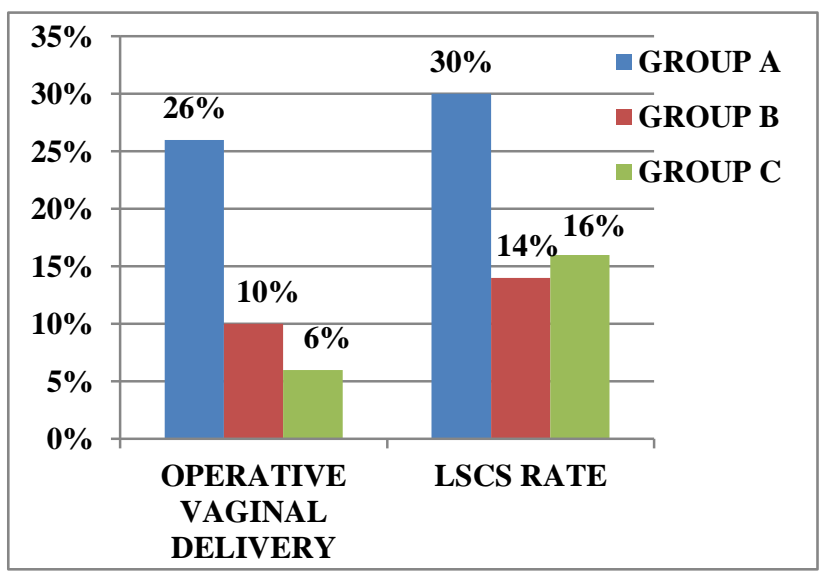

Figure 4: Mode of delivery. 
As shown in Figure 4, the rate of operative vaginal delivery was $26 \%$ in group A which was significantly higher compared to group B (10\%) and group C (6\%). Also shown in Figure 4, the rate of caesarean delivery was significantly higher in group A $(30 \%)$ compared to group B (14\%) and group C (16\%).

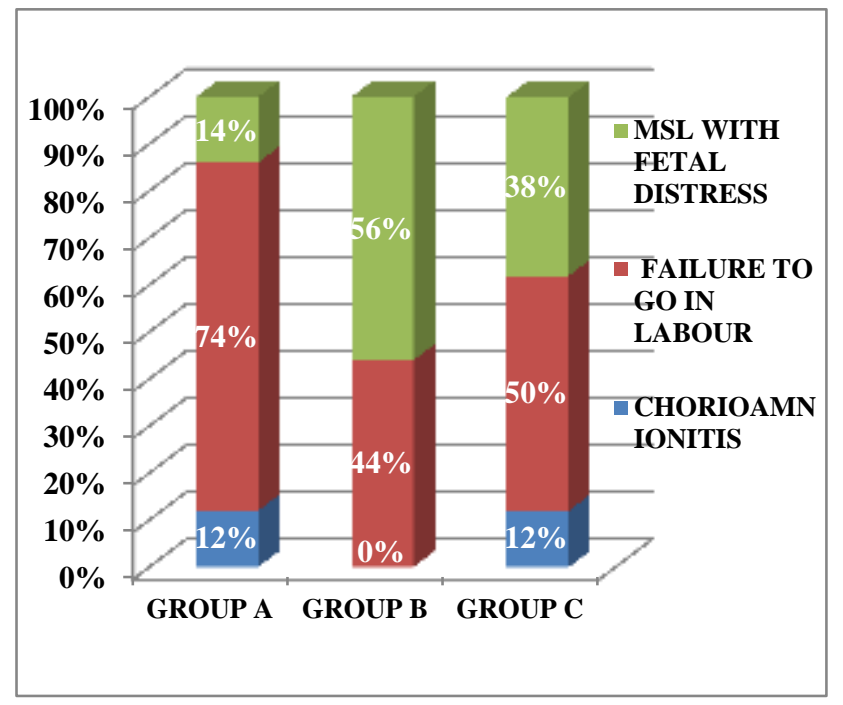

Figure 5: Indications of caesarean section.

As shown in Figure 5, in group A, majority of patients were taken up for caesarean section due to failure to go into labour $(74 \%)$. In group B, $56 \%$ patients were taken up for caesarean section due to meconium stained liquor with fetal distress and $44 \%$ patients were taken up due to failed induction. In group C, 38\% patients were taken up for caesarean section due to meconium stained liquor with fetal distress, $50 \%$ were taken up due to failed induction and $12 \%$ were taken up due to chorioamnionitis.

Rate of neonatal infection was higher in group A (4\%) as compared to group B $(2 \%)$ and group C $(2 \%)$, however the difference was not statistically significant.

\section{DISCUSSION}

Several investigators have compared immediate induction with $25 \mathrm{mcg}$ vaginal misoprostol and immediate or delayed induction with oxytocin in women with PROM at term. Some studies have compared immediate induction with PGE2 gel and delayed induction with oxytocin in women with PROM at term.

The present study was done comparing the 3 groups- 1) expectant management of term PROM 2) immediate induction with misoprostol and 3) immediate induction with dinoprostone gel.

Patients in the 3 groups were comparable with respect to maternal age, gestational age, parity, educational status, and socio-economic status, previous history of PROM and previous history of abortions. All subjects were between
19-28 years of age with mean age of 25 years. This finding correlates with other studies who reported the mean age as 22 years and 25 years. ${ }^{12,13}$ In expectant management, only $30 \%$ women went into spontaneous labour without augmentation which is similar to other studies. $^{1,12}$

Durations of latent and active phase of labour were significantly shorter in induction groups as compared to expectant management group in our study. This finding was similar to the study done by Shanthi et al where PROM-delivery interval was 30.49 hours in expectant group compared to 11.46 hours in active management group. ${ }^{12}$ Study done by Shah et al reported PROMdelivery interval of 13 versus 22 hours in expectant group and induction with PG E2 group. ${ }^{14}$ Ayaz et al reported that induction with PG E1 shortened the PROM delivery interval by 8.7 hours compared to the expectant group. ${ }^{15}$ Choudhuri et al reported interval from rupture of membranes to delivery was significantly shorter in immediate induction group $(\mathrm{P}=0.001)$ compared to delayed induction with oxytocin. ${ }^{1}$

We found presence of meconium stained liquor was higher in patients induced with misoprostol $(22 \%)$ versus expectant management group (14\%) and cerviprime group (12\%) but the difference was not statistically significant. Shah et al also reported that there was no significant difference in meconium staining of liquor in the expectant group compared to early induction with PG E2 group. ${ }^{14}$

In the current study, the rates of operative vaginal delivery and caesarean section were significantly higher in expectant management group compared to the induction groups whereas the rates were comparable in induction with PG E1 or E2. This is similar to a study in which the rate of caesarean section was $48 \%$ in expectant group $(\mathrm{p}=<0.01)$ and $10 \%$ in PG E1 group. ${ }^{15}$ Another study reported higher rate of caesarean section among nulliparous women in the delayed induction group but it was only marginally significant $(28.5 \%$ versus $17.8 \%$, $\mathrm{P}=0.049$ ). They also reported a significantly higher rate of operative vaginal delivery in delayed induction group (14.2\% versus $3.5 \%, \mathrm{P}=0.007){ }^{1}$ This is in contrast to a study in which the rates of operative vaginal delivery and caesarean section were lower in expectant management group12 and another study which reported similar rates of caesarean delivery in expectant management group and induction (PG E2) group. ${ }^{14}$

The maternal and neonatal infection rates were higher in expectant management group than the induction groups in our study but the difference was not statistically significant. This finding was similar to the previous studies. ${ }^{1,12}$ In contrast, another study done by Shah et al found that maternal-neonatal morbidity was higher in expectant group, which can be reduced by limited per vaginal examinations, proper aseptic precautions, and appropriate antibiotic coverage. ${ }^{14}$ 


\section{CONCLUSION}

Induction with PGE1/E2 significantly shortens the PROM-delivery interval and lowers the operative vaginal delivery and caesarean rate as compared to expectant management thus reducing the associated maternal morbidity. There is no significant difference in the obstetric outcome in the inductions done with PG E1 and PG E2. Maternal infection rates are comparable in all the three groups. Neonatal morbidity and mortality is not significantly affected by any of the three management protocols.

Funding: No funding sources

Conflict of interest: None declared

Ethical approval: The study was approved by the Institutional Ethics Committee

\section{REFERENCES}

1. Chaudhuri S, Mitra, Biswas P, Bhattacharyya S. Premature rupture of membranes at term: immediate induction with PGE2 gel compared with delayed induction with oxytocin. J Obstet Gynecol India. 2006;56(3):224-9.

2. Larranaga-Azcarate C, Campo-Molina G, PerezRodri'guez, Ezcurdia-Gurpegui M. Dinoprostone vaginal slow release system compared to expectant management in the active treatment of premature rupture of the membranes at term: impact on maternal and fetal outcome. Acta obstetrica. 2008;87:195-200.

3. Hoffmann RA, Anthony J, Fawcus S. Oral misoprostol versus Placebo in the management of prelabor rupture of membranes at term. Int $\mathrm{J}$ of gynec obstet. 2001;72:215-21.

4. ACOG Committee on practice bulletins-obstetrics. ACOG Practice bulletin no. 107: induction of labor. Obstet Gynecol. 2009;114:386-97.

5. Hannah ME, Ohlsson A, Farine D, Hewson SA, Hodnett ED, Myhr TL, et al. Induction of labor compared with expectant management for labor rupture of membranes at term. TERMPROM Study Group. N Engl J Med. 1996;334:1005-10.
6. Gunn GC, Mishell DR, Morton DG. Premature rupture of fetal membranes. A review. Am J Obstet Gynecol. 1970;106:469-83.

7. Grant JM, Keirse MJNC. Prelabor rupture of the membranes at term. In: Chalmers L, Enkin M, Keirse MJNC, editors. Effective care in pregnancy and child birth. Vol. 2. Child birth. Oxford: Oxford University Press; 1989:112-7.

8. Ezra Y, Cohen RM, Abramov Y, Rojansky N. Prelabor rupture of membranes at term: when to induce labor? Eur J Obstet Gynecol Reprod Biol. 2004;115:23-7.

9. Tarik Y, Zamzami Y. Prelabor rupture of membranes at term in low risk women: induce or wait? Arch Gynecol Obstet. 2006;273:278-82.

10. Tran SH, Cheng YW, Kaimal AJ, Caughey AB. Length of rupture of membranes at term and infectious maternal morbidity. Am J Obstet Gynecol. 2008; 198:700:e1-5.

11. Dare MR, Middleton P, Crowther CA, Flenady VJ, Varatharaju B. Planned early birth versus expectant management (waiting) for prelabor rupture of membranes at term (37 weeks or more). Cochrane Database Syst Rev. 2006;25(1):CD005302.

12. Shanthi K, Devi GP, Bharathi T, Chandrasekharan PA. Comparative study of active versus expectant management and maternal and neonatal outcome in premature rupture of the membranes (PROM) in tertiary care hospital, Tirupathi. IOSR Journal of Dental and Medical Sciences. 2015;14(4):34-9.

13. Varner MW, Galask RP. Conservative management of premature rupture of the membranes. Am J Obstet Gynecol. 1981;140(1):39-45.

14. Shah K, Doshi H. Premature rupture of membrane at term: early induction versus expectant management. Journal of Obstetrics and Gynecology of India. 2012;62(2):172-5.

15. Ayaz A, Saeed S, Farooq MU, Ahmad F, Bahoo LA, Ahmad I. Pre-labor rupture of membranes at term in patients with an unfavorable cervix: active versus conservative management. Taiwan J obstet gynecol. 2008;47:192-6.

Cite this article as: Zutshi V, Bachani S, Goel S, Arora R, Tiwari S. Prelabour rupture of membranes at term prospective study of expectant management versus induction of labour. Int J Basic Clin Pharmacol 2016;5:1408-12. 